\title{
Faecal carriage of group B streptococci
}

\author{
AKMS ISLAM AND ELIZABETH THOMAS* \\ From the Department of Medical Microbiology and the Public Health Laboratory, Whipps Cross \\ Hospital, London E11 1NR, UK
}

SUMMARY Consecutive stool samples from 116 female and 98 male patients (both adults and children), and rectal and vaginal swabs from 28 and 53 cases respectively, were quantitatively cultured for group B streptococci using Islam's medium. Group B streptococcus was recovered from $5 \%$ and $2 \%$ of faeces in female and male patients respectively, and the colony counts ranged from $10^{2}$ to $10^{3} / \mathrm{g}$. In women, the faecal carriage rate was $6 \%$, which was significantly lower than the rectal carriage rate (P 0.02$)$, suggesting that the higher recovery rate $(27 \%)$ from rectal specimens may be due to contamination of swabs by perianal skin flora. Type II group B streptococcus was the only faecal isolate in adults (numbers involved are small for statistical significance), and we suspect that this type strain may be the only resident gut flora in adults, and the gastrointestinal tract is unlikely to serve as the main reservoir of all group B streptococci.

Group B streptococcus appears to be one of the commonest isolates (second only to Escherichia coli) from blood and cerebrospinal fluid of neonates in the first month of life ${ }^{1}$ and has also been incriminated in various infections in adults (particularly in diabetics or in immunodeficient hosts) with isolation from a variety of clinical specimens-blood, CSF, urine, wound swabs, etc. ${ }^{2-5}$

The maternal genital tract appears to serve as the main reservoir of this organism for transmission of infection to neonates. Recently, a few investigators have shown consistently higher recovery rates of group B streptococci from rectal swabs than from any other sites, ${ }^{6-8}$ suggesting that the gastrointestinal tract may be the main reservoir of group B streptococci and that vaginal colonisation may represent contamination from this source, ${ }^{8}$ but actual faecal carriage rates have not been reported before.

This study was undertaken to investigate the faecal carriage rates of group B streptococci in both sexes and to determine whether these bacteria form part of the faecal flora, thus constituting a major source of human streptococcal disease.

\section{Material and methods}

All faecal specimens (from both sexes) submitted to this laboratory over a four-week period were

*Present address: Department of Bacteriology, Royal Liverpool Hospital, Liverpool L7 8XW, UK.

Received for publication 21 February 1980 included in this study. Rectal and high vaginal swabs were also included at a later stage as the stool samples were producing only a few positive results; all swabs were obtained in Stuart's transport medium. The specimens were processed and cultured on the same day within 4 hours of collection except a few swabs which were stored in transport medium at room temperature overnight.

Islam's starch serum agar (SS) medium ${ }^{9}$ was used for quantitative culture for group B streptococci. The single-layered SS agar plates used in this study were made selective with added gentamicin, $6 \mathrm{mg} / \mathrm{l}$, which in preliminary experiments on simulated positive stools/swabs with known concentrations of group B streptococci gave more satisfactory results compared to the media with added gentamicin, $10 \mathrm{mg} / \mathrm{l}$, which inhibited growth of one test strain, and nalidixic acid, which at $15 \mathrm{mg}$ concentration in the media affected pigment production adversely; $8 \mathrm{mg}$ concentration was non-inhibitory to certain coliforms.

For quantitative cultures a faecal suspension of $1 \mathrm{~g}$ in $5 \mathrm{ml}$ nutrient broth was made (for the initial 10 specimens $1 \mathrm{~g}$ of stool on a narrow wooden spatula was weighed for culture, and subsequently an approximate $1 \mathrm{~g}$ sample on a similar spatula was used). The rectal and vaginal swabs were dipped, twirled, and expressed twice (against the container wall) in $1 \mathrm{ml}$ nutrient broth in order to extract the organisms adhering to the swab. The resulting expressed swabs and faecal suspensions in nutrient broth were quantitatively cultured for group B 
streptococci by a surface viable count method (Miles and Misra) using SS agar with gentamicin. As a control, duplicate cultures were done on blood overlayered SS agar plates to assess viable counts in SS agar with gentamicin. The count was expressed as no. of organisms $/ \mathrm{ml}$ of expressed swabs, and $/ \mathrm{g}$ of faeces (wet wt) respectively. Finally, $1 \mathrm{~g}$ of stool (approximately) and $0.5 \mathrm{ml}$ of expressed rectal and vaginal swabs in nutrient broth were inoculated into $5 \mathrm{ml}$ selective Todd-Hewitt broth with added gentamicin $6 \mathrm{mg}$ and nalidixic acid $15 \mathrm{mg} / \mathrm{l}$. Two loopfuls of nutrient broth faecal suspensions and expressed rectal and vaginal swabs in broth using a standard loop $(0.005 \mathrm{ml})$ were also spread on both the SS agar and MacConkey's plates to compare the isolation rates from the direct plating on SS agar, with or without gentamicin, and Todd-Hewitt broth after subculture. The MacConkey's plate served as a safeguard for recovering a group B streptococcal isolate in case SS agar plates should be swarmed by a Proteus species.

The Todd-Hewitt broth cultures were subcultured on blood overlayered SS agar plates after an overnight incubation at $37^{\circ} \mathrm{C}$. All the plates except MacConkey's were incubated anaerobically using a Gas-Pak system. The SS agar plates were examined for orange-red pigmented, and haemolytic (on overlayered plates) colonies after an overnight incubation. Any colony (of Gram-positive cocci) showing orange-red pigments was presumed to be group B streptococcus and was confirmed by Lancefield's grouping. Repeat viable counts using $0.1 \mathrm{ml}$ neat (of stored specimens at $4^{\circ} \mathrm{C}$ ) as an inoculum were done on specimens which yielded positive results only in selective broth.

All group B streptococcal isolates from faeces were serotyped at the Streptococcal Reference Laboratory, Colindale.

\section{Results}

Table 1 shows the recovery rates of group B streptococcus in faeces and rectal and vaginal swabs. Six out of $116(5 \%)$ stool samples from females and two of $98(2 \%)$ from males yielded group B streptococcus. In women, the faecal carriage rate of $6 \%$ was significantly lower than the rectal carriage rate of $27 \%\left(\chi^{2}=5 \cdot 41, P 0 \cdot 02\right)$. The viable counts in eight positive stools ranged from $10^{2}$ to $3 \times 10^{3} / \mathrm{g}$, but the concentration of group B streptococcus in rectal and vaginal swabs appeared to be comparatively higher, ranging between $7 \times 10^{1}$ and $10^{8} / \mathrm{ml}$ of expressed swabs, about half of which (6/13) had a count of $\geqslant 10^{5}$.

The serotyping results of the faecal isolates are shown in Table 2. The type II strains appeared to be the only isolates from adult stool specimens (both sexes), whereas in children these were either type I or III.

Table 1 Prevalence of group B streptococci in stool and rectal and vaginal swabs

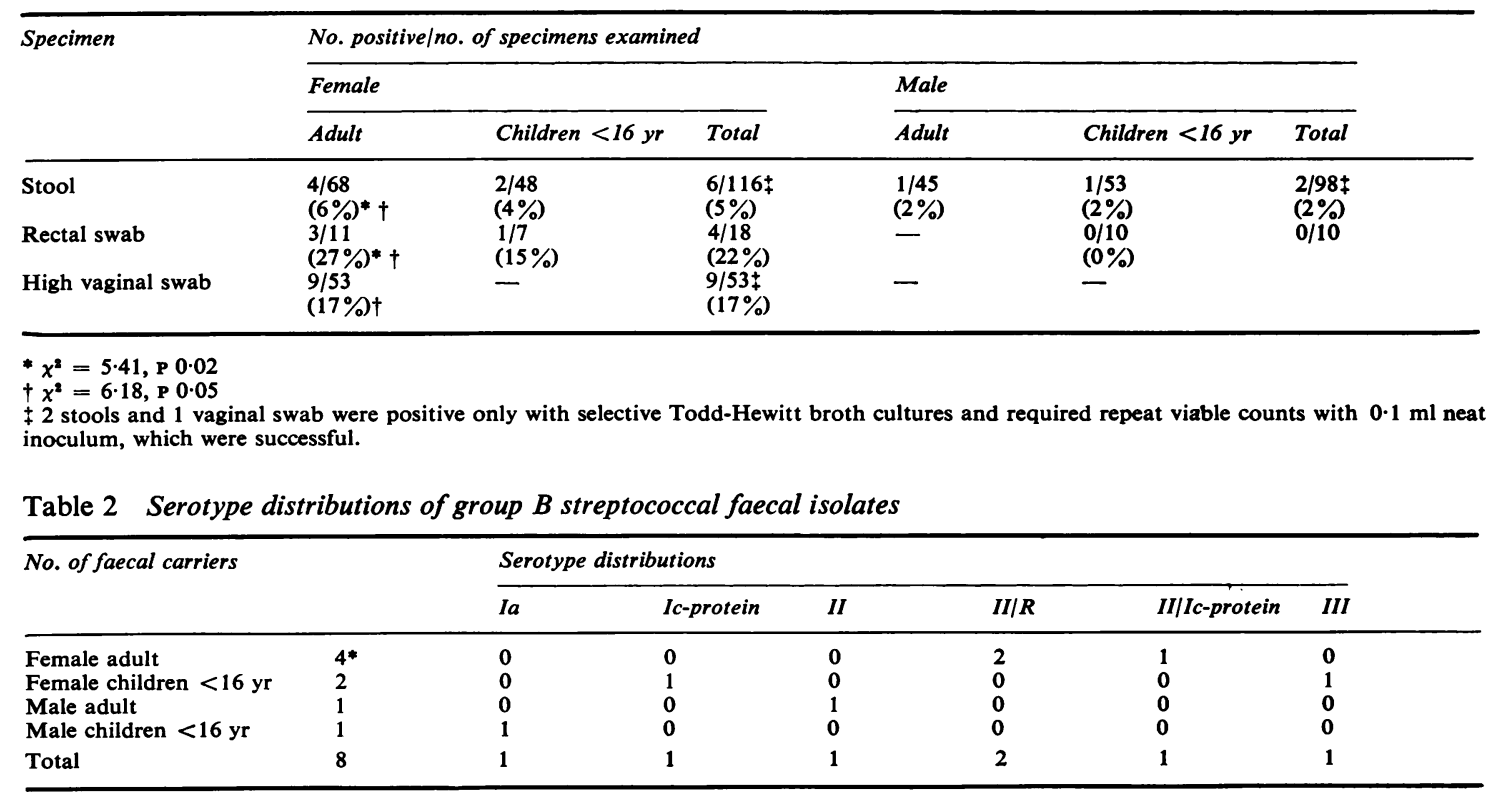

* One isolate died before serotyping could be done. 
The use of selective Todd-Hewitt broth produced more positive results than the direct cultures. Three specimens ( 2 stools and 1 vaginal swab) containing $\leqslant 100$ viable organisms were positive only with selective Todd-Hewitt broth cultures. The SS agar medium with or without gentamicin gave very satisfactory results and appeared almost as sensitive as Todd-Hewitt broth for vaginal swabs. All group B isolates in this study with a presumptive identification on SS agar were confirmed by Lancefield's grouping except one faecal isolate of Streptococcus faecium (group D) from Todd-Hewitt broth subculture, which produced a very faint yellowish pigment on SS agar.

\section{Discussion}

In this study the isolation rates of group B streptococcus from faeces were $5 \%$ and $2 \%$ in females and males respectively. The rectal carriage rates tended to be higher than the vaginal, but we were surprised to find that only 4 of 68 stools $(6 \%)$ in women yielded a positive result, which was significantly lower than the rectal and vaginal isolation rates (Table 1). Moreover, the colony counts in faeces were consistently lower than those in rectal or vaginal swabs. These data indicated that all group B streptococcal isolates from rectal cultures should not be presumed to be of faecal origin. Many of the positive results could have occurred as a result of contamination of swabs by perianal skin flora. The possibility of colonisation of rectum or anal canal locally could not be ruled out altogether, but it seems improbable that the stool could have passed through these passages without being contaminated and thus remained undetected. It would, therefore, appear that perhaps only a proportion of group B streptococcal isolates actually originates from the gut, which is contrary to an earlier suggestion incriminating the gastrointestinal tract as the primary reservoir of all group $B$ streptococci8; the female genital tract in all probability still appears to be the major reservoir of these bacteria. However, recent evidence suggests that the upper respiratory tract $^{10}$ and the male urethra ${ }^{11}$ may also serve as carrier sites.

It would be of interest to note that all the group B streptococcal isolates from faeces in adults (ages 18-62 yr) were type II, whereas in three children $(6 / 12,5$, and $15 \mathrm{yr})$ these were type I or III (Table 2), but the numbers involved are too small to suggest that only the type II group B streptococcus is a resident gut flora in adults. However, the type II strains have been isolated more frequently from the rectum than from the vagina $;^{8}$ and in a very recent study by one of us (report in preparation) we found
$5 \%$ type II perianal/genital carriers among 101 pregnant women. The figure is almost identical with a $6 \%$ faecal carriage rate in women in the present series. Moreover, in three cases type II colonisations were restricted to the perianal and perineal sites only and did not extend up to the vulva or vagina, suggesting that the type JJ strains colonise perianal sites from the faecal source and then spread to the genital site. Conversely, none of the other serotypes (I, III, $R$, and $X$ ) from the remaining 16 positive cases was recovered exclusively from the perianal and/or perineal sites.

On the whole, it appears that with such a low concentration in faeces in a small proportion of women, the gastrointestinal tract is unlikely to serve as a major source and probably plays an insignificant role in the epidemiology of group B streptococcal disease in neonates. The very infrequent isolation of type II strains from serious neonatal infections (unpublished, PHLS Commun Dis Rep) rather confirms our findings.

We thank Dr MT Parker, Streptococcal Reference Laboratory, Colindale, for serotyping our group B streptococcal isolates, and Winthrop Laboratories for supplies of nalidixic acid.

\section{References}

${ }^{1}$ Editorial. Group B streptococci in the newborn. Lancet 1977;1:520-1.

${ }^{2}$ Eickhoff TC, Klein JO, Daly AK, Ingall D, Finland M. Neonatal sepsis and other infections due to group B beta-haemolytic streptococci. $N$ Eng J Med 1964;271: 1221-8.

${ }^{3}$ Butter MNW, DeMoor CE. Streptococcus agalactiae as a cause of meningitis in the newborn, and of bacteraemia in adults. Antonie van Leeuwenhoek 1967;33:439-50.

${ }^{4}$ Mhalu FS. Infection with Streptococcus agalactiae in a London hospital. J Clin Pathol 1976;29:309-12.

5 Parker MT, Ball LC. Streptococci and aerococci associated with systemic infection in man. J Med Microbiol 1976; 9:275-302.

- Franciosi RA, Knostman JD, Zimmerman RA. Group B streptococcal neonatal and infant infections. $J$ Pediatr 1973;82:707-18.

' Mitchell RG, Guillebaud J, Day DG. Group B streptococci in women fitted with intrauterine devices. $J$ Clin Pathol 1977;30:1021-4.

${ }^{8}$ Badri MS, Zawanch S, Cruz AC, et al. Rectal colonization with group B streptococcus: relation to vaginal colonization of pregnant women. $J$ Infect Dis 1977;135:308-12.

- Islam AKMS. Rapid recognition of group B streptococci. Lancet $1977 ; 1: 256-7$.

${ }^{10}$ Ferrieri P, Blair LL. Pharyngeal carriage of group B streptococci: Detection by three methods. $J$ Clin Microbiol 1977;6:136-9.

${ }^{11}$ Manuel FR, MacDonald SW, Embil JA. Prevalence of group B beta-haemolytic streptococci in male urethra. Scand J Infect Dis 1980;12:33-5.

Requests for reprints to: Dr AKMS Islam, Department of Microbiology, Whipps Cross Hospital, London E11 1NR, UK. 